\title{
Usos problemáticos del teléfono móvil: Diferencias entre hombres y mujeres adolescentes de Mérida, Yucatán
}

\section{Problematic use of the mobile phone: Differences between men and women from 12 to 17 years old in Merida, Yucatan}

\author{
Estefanny Hernández \\ Universidad Autónoma de Yucatán \\ Mérida, México \\ estefannyhernandezpuc@gmail.com \\ Orcid: 0000-0003-3369-5876 \\ Vianey Rivero \\ Universidad Autónoma de Yucatán \\ Mérida, México \\ vianeyarp.18@gmail.com \\ Orcid: 0000-0002-3854-5116
}

\author{
Fabiola Morales \\ Universidad Autónoma de Yucatán \\ Mérida, México \\ fabiolamoralesxool@gmail.com \\ Orcid: 0000-0003-4593-3050
}

\author{
Jahaira Uc \\ Universidad Autónoma de Yucatán \\ Mérida, México \\ jahairamarisol.uccastillo@gmail.com \\ Orcid: 0000-0001-8103-4939
}

\begin{abstract}
Resumen-El teléfono móvil es uno de los aparatos tecnológicos mejor aceptados por la población juvenil, por este motivo, el objetivo de la presente investigación es describir las diferencias del uso problemático del teléfono móvil entre hombres y mujeres de 12 a 17 años de Mérida, Yucatán. La muestra incluye a 300 adolescentes que fueron seleccionados de forma aleatoria de distintas escuelas, de los cuales se requerían 150 estudiantes a nivel secundaria y 150 nivel preparatoria, ambos cumpliendo con la edad solicitada. Los resultados se obtuvieron a partir de la aplicación del test titulado: "PMPUQ: Problematic Mobile Phone Use Questionnaire" que mide dimensiones tales como uso prohibido, uso que provoca problemas financieros y el uso que provoca síntomas relacionados con dependencia. Al recabar y comparar la información obtenida, los resultados señalaron diferencias entre ambos sexos, en usos e intereses. De la misma manera se halló que debido a su uso incorrecto, el riesgo a volverse dependientes del teléfono móvil se incrementa cada vez más.
\end{abstract}

Palabras Clave: Teléfono móvil, usos problemáticos, adolescentes, tecnología.

\begin{abstract}
The mobile phone is one of the best accepted technological devices by the youth population, for this reason, the objective of this investigation is to describe the differences in the problematic use of the mobile phone between men and women from 12 to 17 years old in Merida, Yucatan. The sample includes 300 teenagers who were randomly selected from different schools, of which 150 students were required at the secondary level and 150 at the high school level, both meeting the required age. The results were obtained from the application of the test entitled: "PMPUQ: Problematic Mobile Phone Use Questionnaire" which measures dimensions such as prohibited use, use that causes financial problems and use that causes dependency-related symptoms. When collecting and comparing the obtained information, the results pointed out differences between both sexes, in uses and interests. Similarly, it was found that due to misuse, the risk of becoming dependent on the mobile phone is increasing.
\end{abstract}

Keywords: Mobile phone, problematic uses, teenagers, technology.

Sumario: I Introducción, II Usos problemáticos del teléfono móvil, III Metodología, IV Resultados, V Conclusiones. Como citar: Hernández. Estefanny., Morales, Fabiola., Rivero, Vianey., Uc, Jahaira. (2020). Usos problemáticos del teléfono móvil: Diferencias entre hombres y mujeres adolescentes de Mérida, Yucatán. Revista Tecnológica - Espol 32(1). Recuperado a partir de http://www.rte.espol.edu.ec/index.php/tecnologica/article/view/674 


\section{INTRODUCCIÓN}

El teléfono móvil actualmente forma parte de uno de los elementos más indispensables de la comunicación, revolucionando la industria de las telecomunicaciones, desde que se incorporó al ámbito comercial a principios de los 80; la industria del teléfono móvil ha ido creciendo de forma acelerada, especialmente en la década de los $90 \mathrm{y}$ primeros años del siglo XXI, periodo donde su crecimiento ha sido espectacular [18].

Hoy en día es capaz de brindar servicios como datos, audio, video, enviar mensajes, hablar, despertador, fotos, escuchar música, agenda, jugar, grabar videos, navegar en páginas web, chatear y revisar el mail [19]. Lo que ha hecho que se convierta en una herramienta indispensable para las personas, haciéndolas sentir más seguras y productivas [19].

La evolución del uso del teléfono móvil cada vez es mayor. Para el año 1990 existían 11 millones de teléfonos celulares en todo el mundo y para el final de la década la cifra había cambiado a 400 millones; para el año 2004 el número de celulares en uso era de 1,000 millones, excediendo el número de teléfonos fijos [17].

El corto ciclo de vida es una de las principales características, esto conlleva a que evolucionen con gran rapidez, adaptándose a las nuevas necesidades de cada uno de los usuarios. Para separar las etapas que ha tenido la evolución del teléfono móvil se han categorizado generaciones: $1 \mathrm{G}, 2 \mathrm{G}, 3 \mathrm{G}, 4 \mathrm{G}, 5 \mathrm{G}$, habiendo cada una impactado en el servicio de las telecomunicaciones, privatizando empresas y afectando la economía, lo que forzó el surgimiento de nuevas empresas ofreciendo diferentes opciones al cliente para su comunicación, como la distancia, el costo, internet, entre otros [19].

Los adolescentes se han convertido en los principales usuarios de los diferentes servicios que ofrece el teléfono móvil; es parte normal de su cotidianeidad, y lo usan para construir un vínculo y como un medio para organizarse y definir el propio espacio en relación con otros. Se ha señalado que el rango de edad más vulnerable es entre 11 y 18 años [13].

Otro punto importante que señalar es el término "uso problemático" el cual se ha elegido en lugar de "adictivo" [10], dada la falta de consenso en torno a la terminología relativa a las "adicciones tecnológicas" [2]. Entre los usos problemáticos en los cuales se indagó son: dependencia, problemas financieros y usos prohibidos.

\section{USOS PROBLÉMATICOS DEL TELÉFONO MÓVIL}

El uso del teléfono móvil, en adolescentes, constituye un medio de socialización, pero a su vez, da lugar para amenazas y conductas violentas, creando una forma de maltrato digital.

Con el paso del tiempo es cada vez más notoria la creciente disponibilidad en la población juvenil, así como en niños de todas las clases sociales y edades.

El uso problemático del teléfono móvil es en función de la edad, ya que se considera que en la adolescencia se da con mayor intensidad y se normaliza con la edad para convertirse en un uso más profesional y con menor carácter lúdico, debido a los intereses con el paso del tiempo [12].
Los adolescentes, como ya se había mencionado, son los principales usuarios de los diferentes servicios que ofrece la telefonía móvil o los que dedican cada vez más tiempo y recursos económicos. El uso del teléfono se ha convertido en una necesidad y en una herramienta habitual de la comunicación, especialmente en la población joven, ya que, en la actualidad, forma parte de sus vidas, representa modernos instrumentos de socialización y se ha convertido en una herramienta habitual de comunicación [13].

Según [1] existen varias razones por las cuales el teléfono móvil resulta atractivo para los adolescentes; una es el tipo de comunicación que éste les permite, es decir, un contacto continuo e inmediato de tal manera que pueden mantener y expandir sus relaciones sociales. Así como el tiempo y la privacidad es atemporal, se pueden conectar a cualquier hora del día o de la noche, aunque la "privacidad" está sobrevalorada. Igualmente, respecto a la identidad personal, el móvil permite el grado de personalización y facilita el proceso de identidad, y otra razón es el estatus social que este otorga.

\section{A. Dependencia}

Debido al incremento de las nuevas tecnologías, el uso del teléfono móvil en adolescentes en los últimos años ha aumentado significativamente y con esto la dependencia a éstos también se ha visto incrementada [11].

Entendiendo la dependencia como un estado en el que una persona necesita un determinado estímulo para lograr una sensación de bienestar [2], podemos observar cómo la dependencia es un fenómeno de preocupación pues en muchas ocasiones el adolescente no se da cuenta de la dependencia que el móvil le ha generado y, por esta razón, el problema va en aumento [11].

La etapa de dependencia suele ser muy compleja ya que una vez iniciado este proceso es muy difícil de detener, por lo que el individuo llega a sentirse desbordado e incapaz de admitir lo que le sucede. Diferentes estudios sostienen que la preocupación social por posibles malos usos del móvil ha descendido, fundamentalmente por la disminución en las facturas que pagan las familias; y debido a esto la preocupación por el aumento de los malos hábitos en el empleo del teléfono móvil es mayor, pues al disminuir el costo de las facturas, el uso del móvil se ve incrementado y con esto la dependencia [11], así es cuando el teléfono móvil comienza a acarrear problemas para el individuo y su entorno.

Un estudio realizado en el año 2012 trataba el grado de dependencia a la telefonía móvil; los resultados indicaron que el $4 \%$ de los estudiantes son muy dependientes, el $57 \%$ son dependientes y el $39 \%$ de los participantes no tuvo ninguna dependencia [3].

De acuerdo con un estudio que se realizó con el Test de Dependencia del Móvil (TDM), éste arrojó resultados donde las mujeres presentan mayor dependencia que los hombres. De igual manera se encontró que, en un estudio realizado en el año 2016 en cuanto a la dependencia en el empleo del móvil, se corrobora que las chicas mostraban una mayor dependencia en su empleo y manifestaban su inquietud en caso de no tener acceso a los mismos, generando estas actitudes conflictivas frecuentes con sus círculos cercanos [11]. 


\section{B. Uso Prohibido}

La falta de control sobre el uso del teléfono móvil se ha reflejado en la frecuencia y en el tiempo invertido, esto ha afectado de manera considerable el rendimiento y la eficacia de las personas, en este caso los jóvenes, en su día a día. En los últimos años numerosos estudios han evaluado el tiempo invertido, entre ellos, el estudio internacional Health Behavior in School-aged Children (HBSC) realizado en 42 países de Europa y América del Norte mostró que el $63 \%$ de los adolescentes excedía las recomendaciones de tiempo en el móvil; así mismo, la Encuesta Nacional de Salud en España señaló que el 52.3\% de los niños y adolescentes pasaban entre semana más de 2 horas de ocio en el teléfono móvil; la encuesta concluyó que los adolescentes no cumplen las recomendaciones que se les puedan dar sobre el tiempo de pantalla y éste ha ido en aumento hasta un $72.8 \%$ [16]. Es por esta falta de autocontrol que, su inserción, no siempre esté siendo armoniosa en el conjunto de las prácticas y las situaciones colectivas; existen lugares donde se tiene prohibido el uso de éste. Así, mientras en el ámbito de la familia o el laboral el acostumbramiento ha sido el modo de incorporación del teléfono celular, en el espacio educativo su inserción ha generado tensiones singulares, sea porque los jóvenes parecen no entender el sentido de la pertinencia en el uso social o sea porque se asume que toda innovación tecnológica debe transitar siguiendo una trayectoria de embudo, y siempre bajo la supervisión institucional. En el ámbito escolar hay una tendencia generalizada a prohibir su uso, a sancionar a los estudiantes y a descalificar anticipadamente al celular como un recurso para la actividad educativa [14]; actualmente se ha tratado de sensibilizar a la población acerca de no manejar y utilizar el teléfono móvil al mismo tiempo, ya que se ha realizado un número considerable de investigaciones que señalan que constituye un gran peligro en las vías, tanto para conductores como para transeúntes, lo cual convierte el automóvil en otro lugar prohibido [15].

En la etapa de la adolescencia especialmente, en donde las personas suelen ser más vulnerables, deciden ignorar varios señalamientos sobre la prohibición de algunas cosas, como lo es la prohibición del uso del teléfono móvil; esto con el tiempo alimenta la dependencia de la persona y conforme esta conducta perdure será más difícil erradicar los usos problemáticos [10].

\section{Problemas Económicos}

En la última década el teléfono móvil pasó de ser un objeto exclusivo a cierta parte de la población, como lo serían los profesionales, para extenderse a prácticamente toda la población. Las múltiples funciones de las que hoy en día se dispone, gracias al desarrollo tecnológico, lo han convertido en uno de los bienes de mayor consumo [2].

En cuanto a adolescentes se habla, es uno de los productos que más aprecian y anhelan; para ellos, elegir un teléfono no es cuestión de comunicación, funciones, relación calidad/precio [2]; sin embargo, en cuestiones de género, como se vio previamente, estudios han puesto de manifiesto que son las mujeres las que hacen un mayor uso y por lo tanto un mayor consumo. Entre los 12 y 14 años de edad el gasto es de aproximadamente $\$ 366.00$ y entre los 17 y 18 años es $\$ 512.93$. Si cuentan con modalidad de prepago, el gasto total se encuentra entre los $\$ 300.00 \mathrm{y}$ $\$ 600.00$ pesos mensuales [2].

Como se vio, ahora la cantidad que se invierte suele ser mayor a lo que realmente se dispone, recortando gastos de otras cosas que pueden llegar a ser fundamentales para priorizar el uso del móvil.

Otro punto por resaltar es que las mujeres han reportado tener más problemas con sus familiares a causa del gasto económico del teléfono móvil, ya sea que se lo pagaban ellas como si lo hacían los padres [2].

Las compañías telefónicas saben la importancia que la parte económica juega en el teléfono móvil y ha diseñado programas para facilitar este servicio, de tal manera que prestan sus servicios por medio de contratos, en los cuales le permiten al consumidor tener acceso a todas las funciones sin problemas de corte por falta de pago; eventualmente le llegaran facturas que tendrá que ir pagando, lo cual ha perpetuado un consumo menos responsable [10].

\section{METODOLOGÍA \\ A. Enfoque de Investigación}

El enfoque que se le dará a esta investigación será cuantitativo ya que se pretende buscar resultados desde una perspectiva externa y objetiva. La intención de este enfoque es buscar la exactitud de mediciones o indicadores sociales con el fin de generalizar sus resultados a poblaciones o situaciones amplias.

\section{B. Tipo de Estudio, Método y Diseño}

El tipo de estudio que se utilizará es de dos muestras independientes; las muestras son independientes cuando no existe relación entre los sujetos de una u otra, lo cual se puede garantizar si los sujetos son asignados aleatoriamente a cada una de las muestras.

Es por ello, por lo que se busca saber si hay alguna relación entre el uso problemático del teléfono móvil y el sexo en los adolescentes entre 12 y 17 años [4]. Esto se consideró ideal para esta investigación ya que se tienen dos variables y este tipo de estudio. "En ocasiones sólo se analiza la relación entre dos variables, pero con frecuencia se ubican en el estudio relaciones entre tres, cuatro o más variables" [4].

El método que se abordará será el de ex post-facto tipo encuesta; el motivo se dio ya que, al no haber manipulación intencional de la variable independiente, pero sí de selección de valores, da como resultado el que no se puedan establecer las relaciones causales [5].

Solo se puede estudiar la relación entre variables que cavarían con el fenómeno estudiado; esto quiere decir que sólo se observará la relación que hay entre el uso problemático del teléfono móvil y el sexo, aunque esto no quiere decir que una sea la causa de la otra.

El diseño que se llevará a cabo en esta investigación es no experimental ya que se realizará sin manipular deliberadamente las variables. Lo que se hará en esta investigación es observar fenómenos tal como se dan en su contexto natural, para posteriormente poder analizarlos. Se considera que este diseño es apropiado para la investigación ya que se desea aplicar a adolescentes que 
están estudiando, entonces los individuos ya pertenecen a un grupo. Otra razón por la que el diseño es no experimental es porque estos estudiantes ya han tenido la influencia del uso del teléfono móvil en sus vidas, por lo que tienen la experiencia y los conocimientos sobre el tema que se abordará; entre eso está, el uso problemático que tienen con el teléfono móvil, por lo tanto esta investigación coincide un punto más con el diseño no experimental, en la cual dice que es sistemática y empírica en la que las variables independientes no se manipulan porque ya han sucedido.

\section{Variables}

\section{1) Uso problemático del teléfono móvil}

Definición operacional: Respuesta al instrumento "Problema con el teléfono móvil. Escala de uso (MPPUS)" y "problemática del uso del teléfono celular (PMPUQ)"

2) Sexo

Definición operacional: Respuesta de los(as) participantes a la pregunta sexo al cual pertenecen.

\section{Instrumento}

El instrumento es una escala tipo Likert (1 "totalmente de acuerdo" a 4 "totalmente desacuerdo") titulado: "PMPUQ: Problematic Mobile Phone Use Questionnaire" [6] basada en la investigación de los autores Billieux, Van der Linden y Rochat, del año 2008; la escala identifica los distintos tipos del uso problemático del teléfono móvil: uso peligroso, uso prohibido, problemas financieros $y$ dependencia. De este instrumento utilizado se eliminó una dimensión, el cual es uso peligroso ya que las afirmaciones constan de aspectos que un adolescente entre 12 y 17 años aún no puede realizar, tales como conducir o necesitar una licencia de conducir ya que todos tienen menos de 18 años y no tienen la edad suficiente para hacer o tener lo antes ya mencionado. De la misma manera se eliminaron 8 ítems, entre los cuales están el i2, i4, i7, i10, i13, i17 e i18, ya que no se encontró relación con la subescala a .20

En el instrumento de PMPUQ se encuentran 30 ítems. El alfa de Cronbach para cada uno de los factores fue de 0.65 para uso prohibido; 0.84 para uso dependiente y 0.85 para problemas económicos. El rango de puntuación es 30120 (a mayor puntuación, mayor uso problemático, potencial y real).

\section{E. Participantes y Muestreo}

La investigación se llevará a cabo con un total de 300 participantes, entre los cuales deberán ser 150 hombres y 150 mujeres que estén cursando actualmente la secundaria y el bachillerato, éstas serán de escuelas públicas, por tal motivo se escogieron las secundarias "Silvia Nelly Cuevas Aguilar" y "Santiago Burgos Brito" al igual que la preparatoria "Conalep Mérida" y "Prepa México" para llevar a cabo la investigación. Para la parte cualitativa la población contara con un total de 12 participantes provenientes de las escuelas anteriormente mencionadas y algunas otras externas a estas instituciones.

Los participantes serán de ambos sexos y uno de los requisitos que deberán cumplir es tener una edad entre los 12 y 17 años y que cuenten con un teléfono móvil.
El muestreo que se utilizó fue no probabilístico aleatorio ya que no estará involucrada la decisión del investigador, igualmente cada miembro tiene la misma probabilidad de ser elegido para formar parte de la muestra y ésta tendrá una lógica al azar [6].

\section{RESULTADOS}

El análisis de los datos se realizó en el programa Statistical Package for the Social Sciences (SPSS) en su versión 24.0. En primer lugar, se realizó un análisis descriptivo a través de la media y desviación estándar si como también un análisis de consistencia interna por medio del coeficiente alfa de Cronbach para la escala y sus respectivos factores uso prohibido, dependencia y problemas financieros.

Un primer paso fue el análisis descriptivo de las dimensiones: uso prohibido, dependencia y problemas financieros. Los datos observados fueron los siguientes y se presentan en la TABLA I

en la dimensión de usos prohibidos se obtuvo una media general de 1.97, y en esta dimensión los hombres obtuvieron una media de 1.93 y las mujeres de 2.02, lo cual indica que las mujeres utilizan en mayor cantidad el móvil en lugares donde no deberían a diferencia de los hombres; en la dimensión de dependencia se obtuvo una media global de 2.61, y la media de hombres en esta dimensión fue de 2.54 y de 2.69 en mujeres, esto indica que las mujeres tienen mayor dependencia hacia el teléfono móvil en comparación a los hombres; y en la dimensión de problemas financieros se obtuvo una media total de 2.76 , y la media de esta dimensión en hombres fue de $2.69 \mathrm{y}$ la media de las mujeres fue de 2.84 , esto quiere decir que las mujeres perciben mayor gasto en el móvil en comparación con los hombres. Ver TABLA I

TABLA I

MEDIAS Y DESVIACIÓN ESTÁNDAR DE LAS DIMENSIONES DE USOS PROBLEMÁTICOS DEL TELÉFONO MÓVIL.

\begin{tabular}{|l|l|l|l|l|l|l|}
\hline \multirow{2}{*}{ Dimensiones } & \multicolumn{2}{|l|}{ Hombres } & \multicolumn{2}{l|}{ Mujeres } & \multicolumn{2}{l|}{ Total } \\
\cline { 2 - 7 } & $\mathbf{M}$ & DS & M & DS & M & DS \\
\hline Usos prohibidos & 1.93 & .66 & 2.01 & .70 & 1.97 & .68 \\
\hline Dependencia & 2.54 & .88 & 2.69 & .83 & 2.61 & .85 \\
\hline $\begin{array}{l}\text { Problemas } \\
\text { financieros }\end{array}$ & 2.69 & .69 & 2.84 & .64 & 2.76 & .66 \\
\hline
\end{tabular}

Seguidamente, se realizó un análisis de correlación entre los factores: uso prohibido, dependencia y problemas financieros; por medio de la prueba estadística Correlación de Pearson, tal como se señala en la TABLA II

Se pudo observar que existe una relación positiva, relativamente baja y significativa entre los usos prohibidos $\mathrm{y}$ los problemas financieros $(\mathrm{r}=.177 ; \mathrm{p}=.000)$, lo cual indica que, a mayor uso del teléfono en lugares prohibidos, aumentaran los problemas financieros. Igualmente, se observó que existe una relación positiva y moderada entre la dependencia y los problemas financieros $(\mathrm{r}=.681 ; \mathrm{p}=$ .002) pero la relación no es estadísticamente significativa, ya que su significancia es mayor a 0,05 ; lo cual indica que mientras más dependiente sea la persona al teléfono móvil 
más problemas financieros obtendrá. Como se muestra en la TABLA II

TABLA II

CORRELACIÓN ENTRE LOS FACTORES USO PROHIBIDO, DEPENDENCIA Y PROBLEMAS FINANCIEROS

\begin{tabular}{|c|c|c|c|c|}
\hline & & $\begin{array}{l}\text { Uso } \\
\text { prohibid } \\
\text { o }\end{array}$ & $\begin{array}{l}\text { Dependenc } \\
\text { ia }\end{array}$ & $\begin{array}{l}\text { Problema } \\
\text { s } \\
\text { financier } \\
\text { os }\end{array}$ \\
\hline $\begin{array}{r}\text { Uso } \\
\text { prohibido }\end{array}$ & $\begin{array}{l}\text { Correlació } \\
\text { n de } \\
\text { Pearson } \\
\text { Sig. } \\
\text { (bilateral) } \\
\text { N }\end{array}$ & $\begin{array}{l}1 \\
300\end{array}$ & $\begin{array}{l}.084 \\
\\
.145 \\
300\end{array}$ & $\begin{array}{l}.177 \\
.002 \\
300\end{array}$ \\
\hline $\begin{array}{r}\text { Dependenc } \\
\text { ia }\end{array}$ & $\begin{array}{l}\text { Correlació } \\
\text { n de } \\
\text { Pearson } \\
\text { Sig. } \\
\text { (bilateral) } \\
\text { N }\end{array}$ & $\begin{array}{l}.084 \\
\\
.145 \\
300\end{array}$ & $\begin{array}{l}1 \\
300\end{array}$ & $\begin{array}{l}.177 * * \\
.002 \\
300\end{array}$ \\
\hline $\begin{array}{l}\text { Problemas } \\
\text { financieros }\end{array}$ & $\begin{array}{l}\text { Correlació } \\
\text { n de } \\
\text { Pearson } \\
\text { Sig. } \\
\text { (bilateral) } \\
\text { N }\end{array}$ & $\begin{array}{l}.177 * * \\
.002 \\
300\end{array}$ & $\begin{array}{l}.681 \\
.000 \\
300\end{array}$ & $\begin{array}{l}1 \\
300\end{array}$ \\
\hline
\end{tabular}

Finalmente, se utilizó la prueba estadística t de Student para observar las diferencias entre los factores de la escala "PMPUQ: Problematic Mobile Phone Use Questionnaire". En la dimensión de usos prohibidos se encontró una t (29) de -.936; con una probabilidad de .350 , la cual indica que la Ho se acepta, en cuanto a la dimensión de dependencia se encontró una $\mathrm{t}$ (29) de -1.569; con una probabilidad de .118 , la cual indica que la Ho se acepta y finalmente en cuanto a la dimensión de problemas financieros se encontró una t (29) de -1.988; con una probabilidad de .048 , la cual indica que la Ho se rechaza mostrando así que si existe usos problemáticos estadísticamente significativos entre los hombres y las mujeres, lo cual se puede observar en la TABLA III

Con esto podemos ver que aun cuando se presenten problemas financieros, no tiene relación con los usos problemáticos y con la dependencia. Ver TABLA III

TABLA III

DIFERENCIAS ENTRE LOS FACTORES USO PROHIBIDO, DEPENDENCIA Y PROBLEMAS FINANCIEROS

\begin{tabular}{|l|l|l|l|}
\hline Dimensiones & t & Gl & P \\
\hline Uso prohibido & -.93 & 29 & .35 \\
\hline Dependencia & -1.56 & 29 & .11 \\
\hline Problemas financieros & -1.98 & 29 & .04 \\
\hline
\end{tabular}

Finalmente, en la TABLA IV, observamos que la media más alta encontrada es en cuanto a la dimensión de problemas financieros respecto al sexo femenino y la dimensión con la media más baja fue la de usos prohibidos en hombres, observando con esto que las mujeres son las que presentan un mayor uso problemático. Ver TABLA IV.

TABLA IV

MEDIAS POR SEXO ENTRE LOS FACTORES USO PROHIBIDO, DEPENDENCIA Y PROBLEMAS FINANCIEROS

\begin{tabular}{|l|l|l|}
\hline \multicolumn{3}{|c|}{ Medias } \\
\hline Dimensiones & Hombres & Mujeres \\
\hline Uso prohibido & 1.93 & 2.01 \\
\hline Dependencia & 2.54 & 2.69 \\
\hline Problemas financieros & 2.69 & 2.84 \\
\hline
\end{tabular}

\section{CONCLUSIONES}

Los resultados obtenidos mostraron una relación entre las dimensiones "uso prohibido y problemas financieros", lo cual indica que a medida que la persona utilice con mayor frecuencia su teléfono móvil en lugares prohibidos, como la biblioteca o una sala de cine, incrementará los gastos económicos del mismo aparato; esto probablemente se derive del tipo de comunicación que el teléfono móvil les provea; es decir, si la comunicación es continua, las personas tienden a usarlo sin importar en donde se encuentre; así mismo, en cuanto al tiempo, las personas tienen la facilidad de usar el teléfono a cualquier hora del día, y en esto se coincide con los autores Agüero, Almeida, Espitia, Flores y Espig en el 2014 [7], donde en un estudio que realizaron concluyeron que las personas realmente sí están informadas y son conscientes de los riesgos que el uso del teléfono móvil conlleva y de las restricciones de su uso en algunos lugares; sin embargo a pesar de esto, de igual manera lo siguen utilizando ya que lo que más le preocupa a la población es mantener un estatus con las personas que los rodean. Igualmente, en un artículo del 2017 a cargo de los autores Simó, Martínez, Ballester y Domínguez [6], mencionaron "Una de las preocupaciones centrales se encontraba en el tiempo que se empleaba y el uso que se realizaba en lugares como hospitales, coches, entre otros". Esto, probablemente ayuda en cierta forma a que los gastos incrementen ya que si la preocupación de la población es mantener cierto estatus y el mismo aparato les proporciona una comunicación fácil y un tiempo ilimitado entonces, esto hace que las personas paguen por ciertos servicios, como las llamadas telefónicas, lo cual coincide con el autor Zambrano que en el 2009 [8] mencionó que, la inversión que las personas hacen en el teléfono móvil ayuda a las empresas a realizar mejoras o actualizaciones en los aparatos tecnológicos, de tal manera que se vuelva atractivo al público, y con esto se incremente el consumo tanto de los teléfonos móviles como de los servicios que se ofrecen para éste, haciendo de esta forma una inversión económica más grande.

Otra relación que se obtuvo fue la dependencia con los problemas financieros; esto indica que a mayor uso del teléfono móvil mayor gasto se hace en el mismo. Una de las causas por las cuales esto puede suceder es por la edad; al encontrarse en la preadolescencia y adolescencia se vuelven más vulnerables a la influencia de estos aparatos al no controlar el uso que le dan al teléfono móvil e imitar 
lo que los demás hacen con el mismo; esto se puede reflejar con el autor Sánchez-Carbonell en el 2007 [1], donde menciona que, el móvil tiene varios significados en la vida de los adolescentes, es parte de la cotidianeidad y lo utilizan como medio para organizar actividades, además de que también es un medio para construir un vínculo social y definir su propio espacio en relación con los otros. Así mismo, como se mencionó en el párrafo anterior, cada actualización realizada al teléfono se vuelve más atractiva y en esta población (12-17 años) resulta más tentador. Según de la Gándara y Álvarez (2008) [9], el mayor riesgo en adolescentes es llegar al punto de ser incapaces de desprenderse del teléfono móvil, aún en situaciones peligrosas.

En función del género cabe resaltar que las mujeres presentan una media más elevada en las dimensiones de dependencia y problemas financieros en comparación con los hombres, lo cual quiere decir, que ellas son las que más utilizan el teléfono móvil y por consiguiente las que más invierten económicamente en él; esto concuerda con un estudio que se realizó con el Test de Dependencia del Móvil (TDM) en el cuál arrojaron resultados de que las mujeres presentan mayor dependencia al móvil que los hombres y que son ellas quienes tienen mayores problemas económicos y familiares como consecuencia del gasto económico que les acarrea el uso del teléfono móvil, por la misma dependencia hacia este aparato. Finalmente, Chóliz [2] mencionaba que, además de la dependencia y los problemas financieros, éstas traen consigo problemas con los padres por el uso y gasto excesivo del teléfono celular, esto podría derivarse del tiempo excesivo dedicado al teléfono móvil, de la prioridad que se le da a este aparato por encima de otras actividades y la poca atención que le daban a las personas que se encontraban con ellos por estar usando el teléfono móvil.

El teléfono móvil en la actualidad es un aparato con mucho auge, sin embargo, debido a su uso incorrecto el riesgo a volverse dependientes del teléfono móvil incrementa; este estudio aporta una actualización de la información, en el cual se observó que aún sigue existiendo diferencia en los usos problemáticos entre hombres y mujeres, pero en comparación con las investigaciones realizadas anteriormente se nota que tanto hombres como mujeres ya se están poniendo a la par, ya que esas diferencias ya no son tan grandes. Es importante seguir investigando estos usos problemáticos porque como se observó, los usos problemáticos que se tienen ahora no son los mismos que se tuvieron en años pasados y posiblemente en un futuro no sean los mismos precisamente por las constantes actualizaciones que se les hacen a estos aparatos.

Una limitación fue la edad de los adolescentes con los que se trabajó, ya que debido a que no contaban con la mayoría de edad esto dificultaba que pudieran contestar los instrumentos porque algunos de los participantes no tenían el permiso de sus padres y esto imposibilitaba su participación.

Es necesario seguir investigando sobre los usos problemáticos de los teléfonos móviles y sus efectos en los adolescentes, ya que como se ha mencionado, los teléfonos móviles mejoran y cada vez más rápido por lo cual los usos que le den igualmente cambian; así mismo, sería de gran utilidad investigar sobre los efectos que estos provocan en la salud de los adolescentes y de los que los rodean.

\section{AGRADECIMIENTOS}

Los autores desean expresar su agradecimiento al supervisor de la investigación el Dr. Carlos David Carrillo Trujillo, por haber apoyado esta investigación de principio a fin y no perder la esperanza.

Igualmente, queremos agradecer a la institución la Facultad de Psicología de la UADY, por tener en consideración la importancia de la investigación en nuestro campo y de darnos la oportunidad de poder aprender de ella en las instalaciones.

Así mismo a nuestras familias y al esfuerzo y empeño que cada miembro propició para la realización de este.

\section{REFERENCIAS}

[1] Sánchez, S. (2 de mayo del 2017). Acceso al teléfono móvil: Uso, abuso (¿y dependencia?) del teléfono móvil en el mundo. [Xataka]. Recuperado de https://www.xataka.com/otros/losocho-mapas-dela-desigualdad-en-el-mundo-hay-mejor-acceso-atelefono-movilque-a-agua-y-electricidad

[2] Chóliz, M., Chóliz, M. C. y Villanueva, V. (2009). Ellas, ellos y su móvil: Uso, abuso (¿y dependencia?) del teléfono móvil en la adolescencia. Revista Española de Drogodependencias, 34 (1), 274-288.

[3] Quispé, I. (2012). Factores Asociados Al Grado De Dependencia A La Telefonía Móvil e Internet Y Su Influencia en el Entorno Social De Los Jóvenes Universitarios En La Universidad Andina Nástor Cáceres Velásquez. Revista Científica De La Uancy, 9 (1), 147-152.

[4] Sampieri, R., Baptista, P., Fernandez, C. (2010). Metodología de la investigación. México: Mc Graw Hill.

[5] Franco, A. (2013). El Uso De La Tecnología: Determinación Del Tiempo Que Los Jóvenes De Entre 12 Y 18 Años Dedican A Los Equipos Tecnológicos.

[6] Ballester, M., Domínguez, A., Martínez, A. y Simó, C. (2017). Instrumentos De Evaluación Del Uso Problemático Del Teléfono Móvil/Smart Phone. Health and Addictions, 17 (1), 5-14.

[7] Agüero, D., Almeida, G., Espig, H., Espitia, M. y Flores, A. (2014). Uso Del Teléfono Celular Como Distractor En La Conducción De Automóviles. Salus, 18 (2), 27-34.

[8] Zambrano, J. (2009). Aprendizaje móvil (M-LEARNING). Inventum, 1 (7), 38-41. (9 de abril de 2008). ¿Qué uso le da al celular? Infobae. Recuperado de https://www.infobae.com/2008/04/09/373920-que-uso-le-da-alcelular/

[9] Álvarez, O. (4 de mayo del 2008). Teléfono móvil, sus usos y su futuro. [Todaysten hoy $\mathrm{y}$ futuro]. Recuperado de https://www.elblogsalmon.com/entorno/telefono-movil-sus-usosy-su-futuro

[10] Pérez, E., Monje, M., \& De León, J. (2012). Adicción o abuso del teléfono móvil. Revisión de la literatura. Adicciones, 24(2), 139152.

[11] Ruiz, J., Sanchez, J. y Trujillo, J. (2016). Utilización de Internet y dependencia a teléfonos móviles en adolescentes. Rev.latinoam.cienc.soc.niñez juv [online]. vol.14, n.2, pp.1357$1369 . \quad$ ISSN 1692-715X http://dx.doi.org/10.11600/1692715x.14232080715.

[12] Beranuy M., Chamarro A., C. Graner, X. Validación de dos escalas breves para evaluar la adicción a Internet y el abuso de móvil, Psicothema, 21 (2) (2009), pp. 480-485

[13] Clemente, C. C., \& López, E. R. (2016). Intervención social con adolescentes: Necesidades y recursos. Trabajo social hoy, (77), 7 23.

[14] Domínguez, A., (2015, abril 19). Prohibir el celular, otro anacronismo de la escuela. Revista Universitaria, Tecnologías, 3, pp. 1-23.

[15] Botina, S., Jaramillo, C., Lopera, A. \& Montaño W. (2015). Prevención y Tratamiento de las adicciones desde lo psicosocial. Colombia: Fundación Universitaria Luis Amigó. 
[16] Sevil, J., Abós, A., Aibar, A., Sanz, M., \& García-González, L. (2018). ¿Se deberían replantear las recomendaciones relativas al uso sedentario del tiempo de pantalla en adolescentes? SPORT TKRevista EuroAmericana De Ciencias Del Deporte, 7(2), 75-82. https://doi.org/10.6018/sportk.343271

[17] Escribano, Á., \& Zaballos, A. G. (2002). Evolución de la estructura de mercado de las telecomunicaciones en España.

[18] Gámez, O, Perdomo, R., Hidalgo, L., Escalona, L., \& Romero, R. (2005). Telefonía móvil celular: origen, evolución, perspectivas. Ciencias Holguín, 11(1), 1-8.

[19] Naranjo, D., Buenaño, D., \& Mejía, I. (2016). Evolución de la tecnología móvil. Camino a 5G. Revista Contribuciones a las Ciencias Sociales. 